\title{
Craniospinal irradiation(CSI) in patients with leptomeningeal metastases: risk-benefit- profile and development of a prognostic score for decision making in the palliative setting
}

\author{
Michal Devecka ${ }^{1 *}$ (D), Marciana Nona Duma ${ }^{1,2}$, Jan J. Wilkens', Severin Kampfer ${ }^{1}$, Kai Joachim Borm',
} Stefan Münch ${ }^{1,3}$, Christoph Straube ${ }^{1}$ and Stephanie E. Combs ${ }^{1,3,4}$

\begin{abstract}
Background: The aim of our study was to assess the feasibility and oncologic outcomes in patients treated with spinal (SI) or craniospinal irradiation (CSI) in patients with leptomeningeal metastases (LM) and to suggest a prognostic score as to which patients are most likely to benefit from this treatment.

Methods: Nineteen patients treated with CSI at our institution were eligible for the study. Demographic data, primary tumor characteristics, outcome and toxicity were assessed retrospectively. The extent of extra-CNS disease was defined by staging CT-scans before the initiation of CSI. Based on outcome parameters a prognostic score was developed for stratification based on patient performance status and tumor staging.

Results: Median follow-up and overall survival (OS) for the whole group was 3.4 months (range 0.5-61.5 months). The median overall survival (OS) for patients with LM from breast cancer was 4.7 months and from NSCLC 3.3 months. The median OS was 7.3 months, 3.3 months and 1.5 months for patients with 0,1 and 2 risk factors according to the proposed prognostic score (KPS $<70$ and the presence of extra-CNS disease) respectively. Nonhematologic toxicities were mild.

Conclusion: CSI demonstrated clinically meaningful survival that is comparable to the reported outcome of intrathecal chemotherapy. A simple scoring system could be used to better select patients for treatment with CSI in this palliative setting. In our opinion, the feasibility of performing CSI with modern radiotherapy techniques with better sparing of healthy tissue gives a further rationale for its use also in the palliative setting.
\end{abstract}

Keywords: Cranio-spinal irradiation, Prognostic score, Leptomeningeal carcinomatosis

\footnotetext{
* Correspondence: michal.devecka@mri.tum.de

${ }^{1}$ Klinikum rechts der Isar, Department of RadiationOncology, Technical

University Munich, Ismaninger Strasse 22, 81675 München, Germany

Full list of author information is available at the end of the article
}

(C) The Author(s). 2020 Open Access This article is licensed under a Creative Commons Attribution 4.0 International License, which permits use, sharing, adaptation, distribution and reproduction in any medium or format, as long as you give appropriate credit to the original author(s) and the source, provide a link to the Creative Commons licence, and indicate if changes were made. The images or other third party material in this article are included in the article's Creative Commons licence, unless indicated otherwise in a credit line to the material. If material is not included in the article's Creative Commons licence and your intended use is not permitted by statutory regulation or exceeds the permitted use, you will need to obtain permission directly from the copyright holder. To view a copy of this licence, visit http://creativecommons.org/licenses/by/4.0/ The Creative Commons Public Domain Dedication waiver (http://creativecommons.org/publicdomain/zero/1.0/) applies to the data made available in this article, unless otherwise stated in a credit line to the data. 


\section{Background}

Tumor spread to the leptomeninges (leptomeningeal metastases (LM)) poses a serious condition which leads to rapid deterioration and is ultimately associated with dismal prognosis. Neoplastic meningitis occurs in 3-5\% of patients with cancer [1]. Around 70\% of LM usually presents in patients with widely-metastatic and progressive cancer, however in 5-10\% LM can be the only manifestation of cancer [2]. Sometimes, LM can be the only presentation of the malignant disease. The most common tumors that present with LM are breast cancer, non-small cell lung cancer (NSCLC), and melanoma $[3,4]$. Further, some lymphomas have a high risk of CNS dissemination [5].

Current National comprehensive cancer network $(\mathrm{NCCN})$ guidelines recommend that patients with good performance status (Karnofsky performance score (KPS) $\geq 60$ ) without major neurological deficits, minimal extraCNS disease, and reasonable systemic options should be treated with induction intrathecal (IT) chemotherapy. In the case of breast cancer primary high dose methotrexate (HD-MTX) can be used. Also, whole brain radiotherapy (WBRT) and radiotherapy (RT) to bulky sites are indicated [6].

European society for medical oncology (ESMO) guidelines recommends consideration of focal RT for circumscribed, symptomatic lesions and WBRT for extensive nodular or symptomatic linear LM. According to ESMO "craniospinal irradiation (CSI) is rarely an option for adult patients with LM from solid cancers because of the risk of bone marrow toxicity, enteritis and mucositis, and the usual co-existence of extra-CNS disease" [7]. Nonetheless, modern radiotherapy techniques such as intensity modulated radiotherapy (IMRT) or proton therapy can reduce the aforementioned toxicities [8-10].

In Germany, the guidelines recommend the use of extended WBRT with the inclusion of the upper two cervical vertebrae and focal RT for bulky disease sites. Multiple factors such as the extent of extracranial disease, and of the LM itself (whether microscopic or macroscopic), patient's symptoms, KPS, and tumor histology need to be assessed as to what treatment (IT or systemic chemotherapy or radiotherapy) should be used [11].

Hence, though CSI is a mainstay of curative treatment in patients with medulloblastoma and primitive neuroectodermal tumor (PNET), as well as in ependymoma and germinoma with LM, CSI is not yet fully recommended for palliative treatment in all patients with LM.

The aim of our study was to assess the feasibility and oncologic outcomes in patients treated with craniospinal irradiation (CSI) in patients with leptomeningeal metastases (LM) and to develop a pragmatic prognostic score to stratify patients in this palliative setting.

\section{Methods}

\section{Study patients}

Nineteen patients [12] were treated with palliatively intended CSI in our institution between 2001 and 2015. CSI was either performed in one treatment course, or as spinal irradiation (SI) in patients who had already undergone WBRT. A small gap between the preceding WBRT treatment fields was made to avoid overdose from the matched spinal treatment field. Demographic data, as well as histology of the primary tumor, are summarized in Table 1. In order to avoid selection bias all patients who received at least one fraction of CSI or SI were included in our analysis, even though in some case the treatment had to be stopped early.

Patients treated before 2007 were simulated on a standard treatment simulator (Simulix Evolution, Nucleotron/Elekta). They were immobilized using a thermoplastic mask and vacuum cushion in prone position. Then a $2 \mathrm{D}$ treatment plan was calculated using 2 standard opposed fields for the brain with two attached dorsal fields for the spine. All other patients from 2007 on were treated with a helical tomotherapy (HT) Hi-Art machine (Accuracy Inc., Madison, WI, USA). For patients treated with helical tomotherapy (HT), CT imaging was performed with a 3-5 mm slice thickness on a standard Siemens CT (Siemens Inc., Erlangen, Germany). Patients were immobilized in the supine position using a vacuum couch and a thermoplastic head mask (BRAINLAB, Munich, Germany). OARs and PTV were delineated according to institutional guidelines either in iPlan (BRAINLAB, Munich, Germany) or in Eclipse (Varian Medical Systems, Palo Alto, CA, USA). The CTV comprised the craniospinal axis including the nerve root areas and was divided in CTV Brain, CTV cervical spine, CTV thoracic spine and CTV lumbosacral spine until the second sacral vertebra.

The CTV to PTV margins were as follows:

1. CTV Brain: $6 \mathrm{~mm}-10 \mathrm{~mm}$ in all directions

2. CTV cervical spine: $6 \mathrm{~mm}-10 \mathrm{~mm}$ in all directions,

3. CTV thoracic spine: $10 \mathrm{~mm}$ anteroposterior and 10-15 mm lateral

4. CTV lumbosacral: $10 \mathrm{~mm}$ anteroposterior and 10-20 mm lateral.

The treatment planning was performed with Tomotherapy Planning Station (Tomotherapy Inc., Madison, USA).

The extent of extra-CNS disease was defined by staging CT-scans before the initiation of the CSI (or SI). We reviewed all available MRI findings and images in order to assess the extent of the CNS disease (macroscopic vs. microscopic). All but one patient with breast 
Table 1 Patients' characteristics $(n=19)$

\begin{tabular}{|c|c|c|}
\hline Median Age (years) & 57.8 & (range 31-80) \\
\hline Median Karnofsky performance index & 70 & (range 40-90) \\
\hline Sex & $(n)$ & (percent) \\
\hline - Male & 8 & 42.1 \\
\hline - Female & 11 & 57.9 \\
\hline Treatment technique & $(n)$ & (percent) \\
\hline$-2 D$ & 3 & 15.8 \\
\hline - HT & 16 & 84.2 \\
\hline Treatment Field & $(n)$ & (percent) \\
\hline$-\mathrm{CSI}$ & 15 & 78.9 \\
\hline$-\mathrm{SI}$ & 4 & 21.1 \\
\hline CNS disease & $(n)$ & (percent) \\
\hline - Macroscopic & 18 & 94.7 \\
\hline - Microscopic & 1 & 5.3 \\
\hline Presence of systemic disease outside of the CNS & $(n)$ & (percent) \\
\hline - Yes & 7 & 36.8 \\
\hline$-\mathrm{No}$ & 11 & 57.9 \\
\hline - Not available & 1 & 5.3 \\
\hline \multicolumn{3}{|l|}{ Primary Diagnosis } \\
\hline - Breast cancer & 5 & 26.3 \\
\hline - NSCLC & 5 & 26.3 \\
\hline - Non-Hodgkin Lymphoma & 3 & 15.9 \\
\hline - Adenocarcinoma of gastro-esophageal junction & 1 & 5.3 \\
\hline - Astrocytoma WHO Grade III & 1 & 5.3 \\
\hline - Gastric carcinoma & 1 & 5.3 \\
\hline - Malignant peripheral nerve sheath tumor & 1 & 5.3 \\
\hline - non-CNS NGGCT & 1 & 5.3 \\
\hline - Sarcomatoid CUP & 1 & 5.3 \\
\hline
\end{tabular}

cancer had positive MRI findings, in one patient the diagnosis was made solely based on spinal tap.

\section{Assessment of the treatment benefit}

The treatment benefit was assessed either clinically (by the improvement in pain, neurological deficits, performance status) or with a proven radiological or CSF response. During the CSI, all patients were seen at least twice a week by a radiation oncologist. First follow up was scheduled in 6 weeks after completion of the treatment and thereafter in 3 months intervals. Follow up consisted of clinical examination and MRI or CSF taps were performed at the discretion of the treating physician.

\section{Statistical methods}

Descriptive statistics were used, to sum up, the demographic and dosimetric information. For survival estimation, the Kaplan-Meier method was used. The overall survival (OS) was calculated from the RT begin to the date of death or censured to the date of the last follow up. Mantel-Cox method was used for the subgroup analysis. All statistical calculations were done in SPSS 23.0 and MS Excel.

\section{Compliance with ethical standards}

This study was approved by the Ethics Committee of the School of Medicine of the Technical University of Munich (number 84/15).

\section{Results}

\section{Treatment}

The median time between primary diagnosis of the disease and the initiation of CSI/SI was 1.16 years (range 0-23.66 years). Ten patients received standard chemotherapy within 3 months prior to the CSI. Three out of these ten patients, received intrathecal chemotherapy (IT) alone. The median total CSI dose was 30.6 Gy 
(range 3.0-36.0 Gy) with a median dose per fraction 1.6 Gy (range 1.5-1.8 Gy). Fourteen patients received an additional boost to the area of the macroscopic tumor, with a total median dose to the boost area of $37.6 \mathrm{~Gy}$ (range 9.0-54.0 Gy). Boost dose per fraction varied between 1.8-3 Gy; one patient had a radiosurgery boost to four cerebral metastases with $16 \mathrm{~Gy}$. No concomitant systemic therapy was given parallel to radiotherapy. All but three patients received dexamethasone during CSI/ SI. Three patients received systemic therapy after the CSI/SI. One with mantel cell lymphoma received treatment with Bortezomib for a short period of time which was stopped due to thrombopenia. Patient with hormone-sensitive breast cancer received anastrozole and was alive without signs of disease progress at last follow-up. Another patient with breast cancer responded first to the treatment but was diagnosed with relapse disease 4 months later and received salvage treatment with liposomal cytarabine. She succumbed to disease 5 months later. None of the patients received small molecules, TKIs or immune checkpoint inhibitors prior to the treatment with CSI or thereafter except for bortezomib as stated above.

\section{Survival and improvement of Symtpoms}

Median follow-up and overall survival (OS) for the whole group was 3.4 months (range 0.5-61.5 months).
The median OS for patients with LM from breast cancer was 4.7 months and from NSCLC 3.3 months.

11 out of 19 patients benefited from the treatment either clinically (by the improvement in pain, neurological deficits, performance status) or with a proven radiological or CSF response. There were four "long-term" survivors: two breast cancer patients surviving for a minimum of 11.3 (alive at last follow-up) and 13 months respectively, one patient with disseminated astrocytoma WHO grade III surviving 16 months (alive at last followup) and one patient with NSCLC surviving for more than 5 years (alive at last follow-up). An example of one of these patients is shown in Fig. 1. In 10 out of 19 patients a neurological benefit or pain reduction was recorded after CSI/SI. One patient had no neurologic symptoms prior to treatment or therafter. In 8 out of 19 patients no improvement was observed (Table 2).

In the subgroup analysis (univariate and multivariate) of our group, performance status and extra-CNS disease were significantly associated with survival. Patients with KPS $\geq 70(p=0.018)$ and no extra-CNS disease $(p=$ 0.032) fared much better than their counterparts (Fig. 2). The RT technique had no significant impact on survival ( $p=0.944$; Fig. 2).

A prognostic score combining the KPS and the presence of extra-CNS disease is suggested to stratify patients with LM (Table 3). In our patients' cohort, the

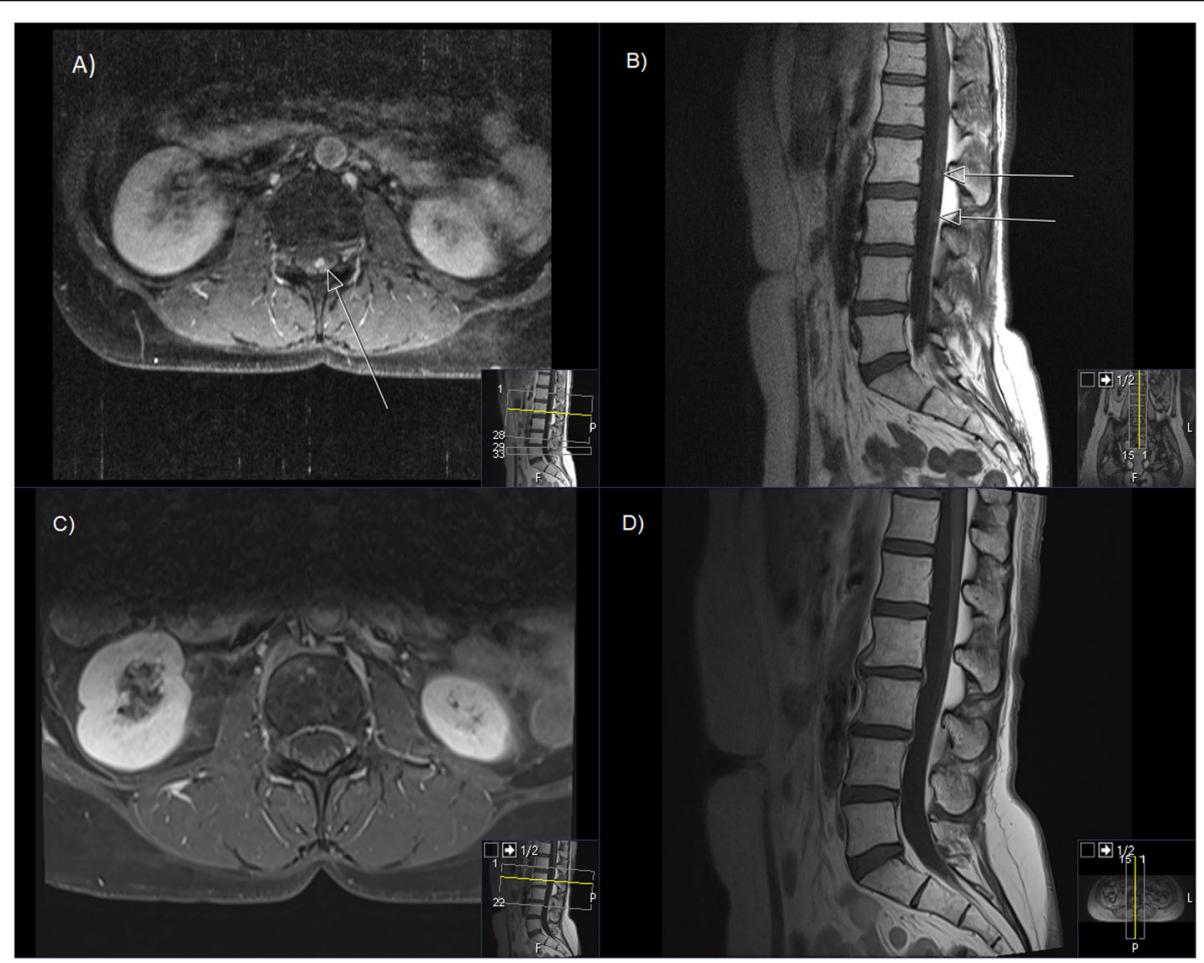

Fig. 1 Example of a patients' MRI before and 6 months after CSI. Contrast enhanced T1 MRI sequence A,B) Prior to CSI, C,D) 6- months post CSI; $A, C)$ axial view $B, D$ ) sagittal view. Arrows point at macroscopic intraspinal disease 
Devecka et al. BMC Cancer $\quad$ (2020) 20:501

Page 5 of 10

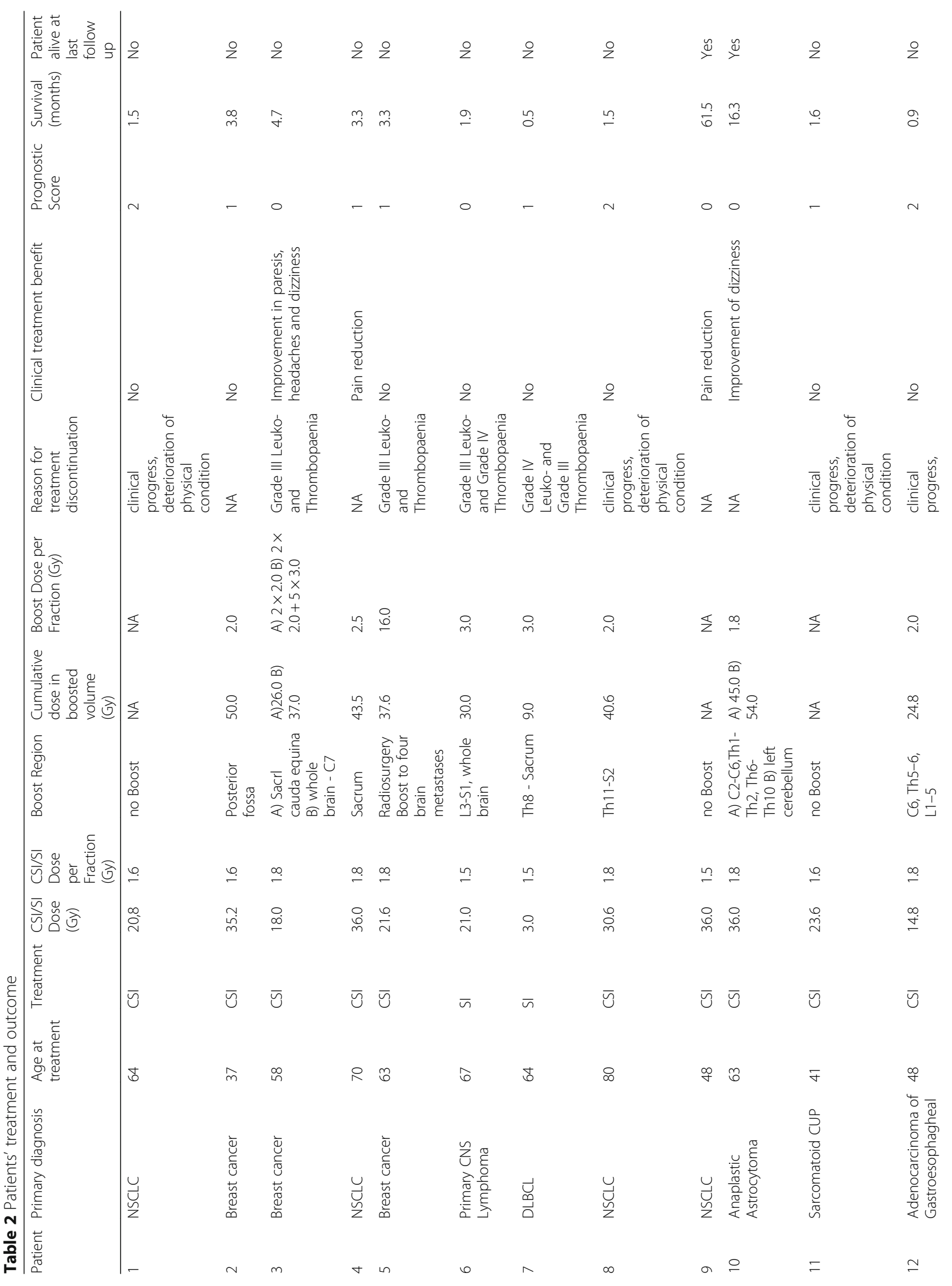


Devecka et al. BMC Cancer $\quad$ (2020) 20:501

Page 6 of 10

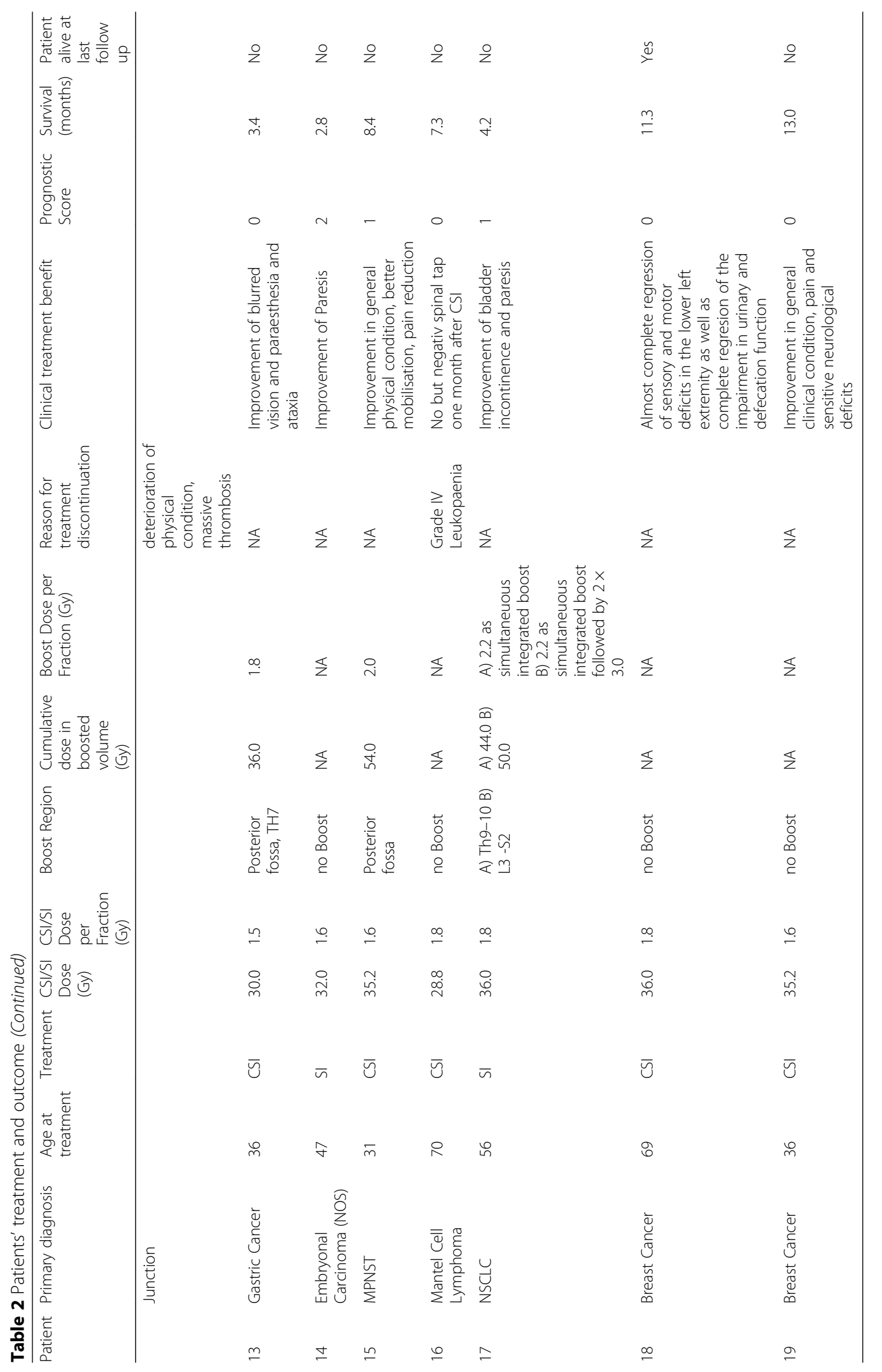



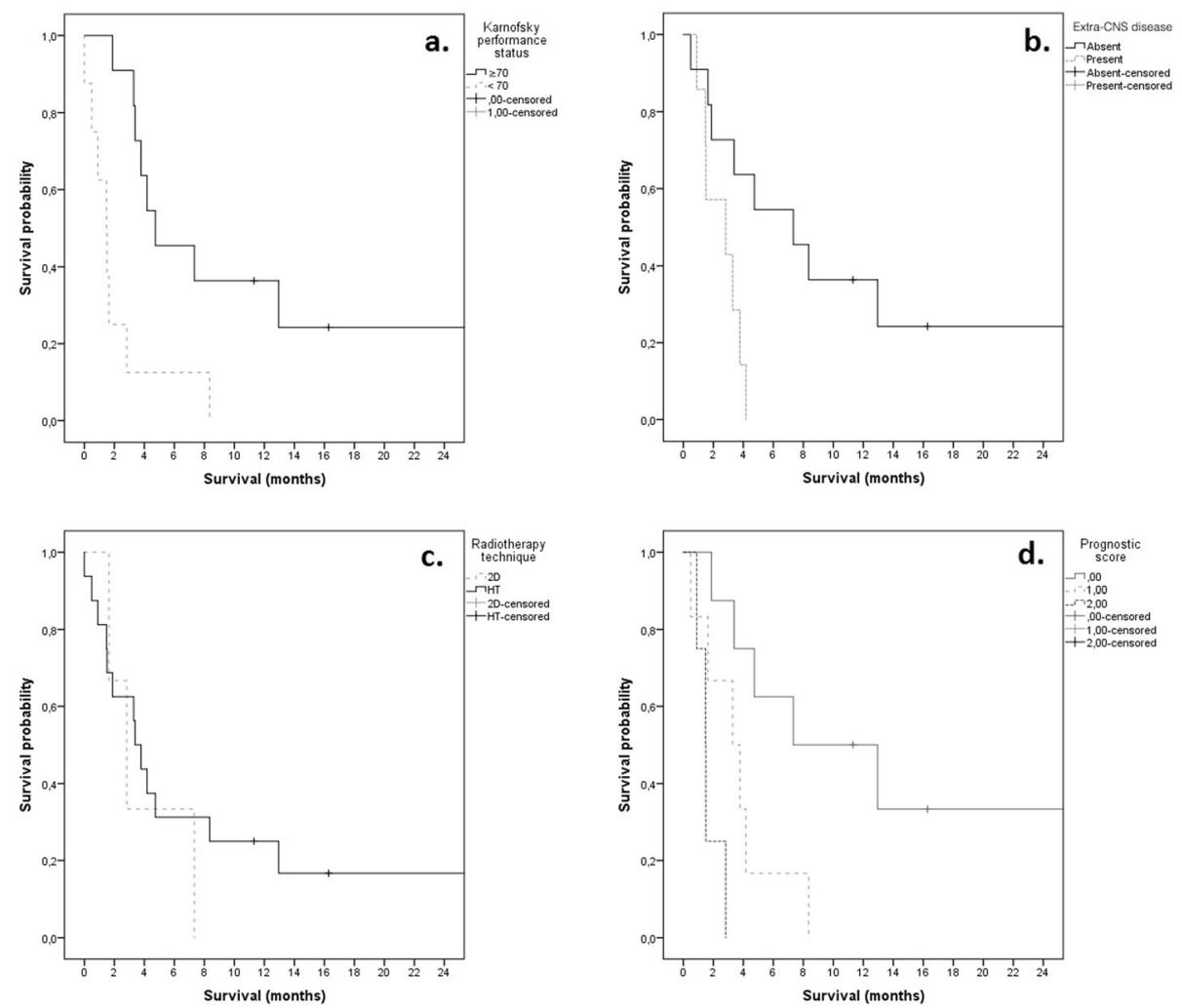

Fig. 2 Survival Probability according to KPS, extra-CNS disease, radiotherapy technique and estimated prognostic score

median OS was 7.3 months, 3.3 months and 1.5 months for patients with 0,1 and 2 risk factors (KPS $<70$ and the presence of extra-CNS disease) respectively. The prognostic score combining these two factors is highly significant $(p=0.001)$ (Fig. 2).

\section{Side effects}

Nine patients did not complete the prescribed treatment. The reasons for treatment discontinuation were mostly grade III-IV cytopenias (5 patients) not recovering after treatment break or general status deterioration with progressive disease in spite of the treatment (4 patients). One patient died of massive thrombosis 1 week after the treatment was discontinued. As his treatment was discountinued due to grade II thrombopaenia (62,000 Tro/ $\mu \mathrm{l})$ this grade $\mathrm{V}$ toxicity was most likely unrelated to the CSI. Hematologic toxicities are summarized in Table 4. Other side effects included xerostomia $(n=3)$, dysgeusia $(n=2)$, candida infection $(\mathrm{n}=2)$, nausea $(n=4)$, vomiting $(n=1)$, mucositis/dysphagia $(n=5)$. All of these side effects were mild (grade I-II).

\section{Discussion}

In our palliative treatment group, the OS of patients receiving CSI due to LM was 3.4 months (14.7 weeks; range $4-267$ weeks). This is in line with survival times reported by the literature on either IT or systemic chemotherapy where median OS ranges between 7 and 30.3 weeks (Table 5).

Focal RT either for bulky spinal disease or WBRT remains a backbone of treatment of patients with LM even though its effect on survival can be questioned [13].

Boogerd et al. showed in a randomized trial that the addition of intrathecal chemotherapy to systemic chemotherapy and focal RT did not provide any additional survival benefit and led to increased toxicity in

Table 3 OS according to prognostic score based on KPS and presence of extra-CNS disease

\begin{tabular}{llll}
\hline Score & & Median OS (months) & Median OS (weeks) \\
\hline 0 & No extra-CNS disease and KPS $\geq 70$ & 7.3 & 31.9 \\
1 & One of the two factors present & 3.3 & 14.3 \\
2 & Extra-CNS disease present and KPS $<70$ & 1.5 & 6.4 \\
\hline
\end{tabular}


Table 4 Hematologic toxicity according to CTCAE v 4.03

\begin{tabular}{llllll}
\hline Grade & 0 & 1 & 2 & 3 & 4 \\
\hline Anemia & $12(63.2 \%)$ & $7(36.8 \%)$ & - & - & - \\
Leukopenia & $3(15.8 \%)$ & $4(21.1 \%)$ & $5(26.3 \%)$ & $5(26.3 \%)$ & $2(10.3 \%)$ \\
Thrombopenia & $1(5.3 \%)$ & $6(31.6 \%)$ & $5(26.3 \%)$ & $6(31.6 \%)$ & $1(5.3 \%)$ \\
\hline
\end{tabular}

patients with LM form breast cancer [14]. Also, in the retrospective study by Oechsle et al. systemic chemotherapy resulted in significantly improved OS as compared to patients treated only with intrathecal chemotherapy, RT or both. Patients who got systemic chemotherapy had a median survival of 24.9 weeks. However, in this study, solid tumors accounted only for $54 \%$ and the rest were hematological malignancies, which normally respond better than solid tumors to treatment with systemic chemotherapy [15]. A number of other reports showed the efficacy of systemic agents (e.g. temozolomide, capecitabine). In some cases even durable responses were shown [13, 16-19].

Pan et al. tested a regimen of intrathecal chemotherapy with MTX and involved field radiotherapy (whole brain or focal to spinal lesions) with $40 \mathrm{~Gy}$ in $2 \mathrm{~Gy} / \mathrm{frac}$ tion. Fifty-nine patients were treated with a median OS of 6.5 months [12].

There is very limited data on the use of CSI as a treatment for patients with LM. To our knowledge, there are only two published study where CSI was evaluated in a palliative setting similar to our study [20, 21].

Hermann et al. studied 16 patients with LM from solid tumors. Nine breast cancer, five NSCLC, one with cancer of unknown primary (CUP) and one renal cell cancer patients were included in his study. The median OS for all patients was 12 weeks (range 4-84 weeks). For patients treated with CSI, only the median OS was 8 weeks.
The median OS was 16 weeks for those treated with IT chemotherapy and CSI. Nonetheless, all patients had synchronous extra-CNS metastases in this study. Improvement in symptoms was seen in 11 patients, two patients had stable disease and in three patients therapy was ended prematurely, due to progressive disease [20]. However, in this study patients were treated without the use of modern RT techniques.

In a recent published study, El Shafie et al. reported on 25 patients treated for LM with the use of HT. The prescribed dose was $36 \mathrm{~Gy}$ in 20 fractions. Sixteen patients had metastatic disease outside of the CNS and 18 had parenchymal brain metastases. Majority of the patients had breast $(n=15)$ or lung cancer $(n=6)$. Median OS from the diagnosis of LM was 19.3 weeks. In multivariate analysis $\mathrm{KPS} \geq 70$, neurologic response and age $<55$ years were prognostic for improved OS [21].

In the analysis of our palliative patient cohort, patients having KPS $\geq 70$ fared also significantly better. For patients with $\mathrm{KPS} \geq 70$, the median OS was 4.7 months (20.6 weeks) compared with 1.5 months (6.6 weeks) for the rest of the group. Further, patients having no extraCNS disease showed significantly improved survival with a median OS for patients with the leptomeningeal disease only of 7.3 months ( 31.9 weeks) as compared to 2.8 months (12.3 weeks) for those also having systemic extra-CNS disease. When these factors were combined a median OS of 7.3 months ( 31.9 weeks) could be achieved for those who had a good KPS and no extra-CNS disease. In those who had one or two adverse factors, the median OS was 3.3 months ( 14.3 weeks) and 1.5 months (6.4 weeks) respectively. These results suggest that cautious patient selection is needed in a palliative setting when considering CSI. Despite the drop-out rate of our patients' collective 11/19 which was much higher than that in the study by Hermann the patients have profited

Table 5 Overview of literature on CSI

\begin{tabular}{|c|c|c|}
\hline Trial & Design & Outcome \\
\hline Grossman et al. & $\begin{array}{l}\text { IT MTX versus thiotepa (59 patients; solid } \\
\text { tumors and lymphoma) }\end{array}$ & OS, 15.9 (MTX) versus 14.1 weeks (thiotepa) \\
\hline Hitchins et al. & $\begin{array}{l}\text { IT MTX versus MTX }+ \text { CYT (44 patients; solid } \\
\text { tumors and lymphoma) }\end{array}$ & OS, 12 (MTX) versus 7 weeks (MTX + CYT) \\
\hline Glantz et al. & LS-CYT versus MTX (61 patients; solid tumors) & OS, 105 (LS-CYT) versus 78 days (MTX), difference not significant \\
\hline Glantz et al. & LS-CYT versus CYT (28 patients; lymphoma) & $\begin{array}{l}\text { OS, } 99.5 \text { (LS-CYT) versus } 63 \text { days (CYT), difference not significant. } \\
\text { Cytologic response rate } 71 \% \text { (LS-CYT) versus 15\% }\end{array}$ \\
\hline Boogerd et al. & $\begin{array}{l}\text { IT versus no IT therapy, but systemic therapy } \\
\text { and RT were given in both arms ( } 35 \text { patients; } \\
\text { breast cancer) }\end{array}$ & OS, 18.3 (IT) versus 30.3 weeks (no IT) \\
\hline \multirow[t]{2}{*}{ Shapiro et al. } & \multirow[t]{2}{*}{ Lymphoma (25 patients) } & $\begin{array}{l}\text { LS-CYT versus all MTX and CYT-treated patients combined: PFS } \\
35 \text { versus } 43 \text { days (not significant) }\end{array}$ \\
\hline & & LS-CYT versus MTX: PFS 35versus 37.5 days (notsignificant) \\
\hline
\end{tabular}

From Leal at al. Abbreviations: IT intrathecal, LM leptomeningeal metastasis, MTX methotrexate, CYT cytarabine, LS-CYT liposomal cytarabine, PFS progression-free survival, RT radiotherapy. (Leal 2011) 
from the treatment either for survival prolongation or for symptom control.

CSI is rarely considered as a treatment option for patients with the leptomeningeal metastatic disease. However, our study together with the study by El Shafie et al. gives a good rationale for offering CSI to well-selected patients. Especially those who have a good performance status, and present with none or controlled extra-CNS disease seem to have the best prognosis after CSI. Although the study by Hermann et al. suggested that patients receiving IT chemotherapy before the CSI fare better, it is somehow counterintuitive first to use IT chemotherapy and to perform radiotherapy after that. If radiation can achieve limited control of macroscopic disease, it is probably moreover so capable of controlling microscopic disease (e.g., the free tumor cells in liquor which could have been affected by the intrathecal chemotherapy). So perhaps intrathecal chemotherapy could be used after prior CSI as "adjuvant" therapy. There are no studies to support this, and there is much fear to apply methotrexate after CSI due to its side effect, but perhaps CSI followed by liposomal cytarabine would be an interesting approach which requires further evaluation.

Historically, two major reasons for the underuse of CSI in patients with LM existed. The concern for acute side effects and technically difficult treatment application. In the Hermann et al. study the major toxicities were myelosuppression (69\%), dysphagia (56\%), mucositis $(44 \%)$ and nausea (19\%). No grading of these toxicities was reported [20]. In the study by El Shafie mild (CTCAE Gr I-II) fatigue and nausea was reported in 84 and $36 \%$ respectively. Skin erythema appeared in $28 \%$ and myelosupression in $32 \%$. Five patients did not complete the prescribed treatment [21]. In our study, the non-hematological toxicities were mild (Grade I-II) and consisted of xerostomia $16 \%$, dysgeusia $10 \%$, nausea $21 \%$ with vomiting in $5 \%$ and mucositis/dysgeusia in $26 \%$ with mucosal candidosis in $10 \%$ patients. Also, most of our patients suffered from hematological toxicity. In our collective, there was a big drop-out rate as $47.4 \%$ of the patients did not complete their prescribed treatment, due to progressive disease or major cytopenia. Nonetheless, with modern radiotherapy techniques CSI can be applied with limited acute non-hematological toxicities $[8,22-25]$. Further, when using helical Tomotherapy (HT), there is no need for field junctions and their daily or weekly shifts as it was the case in the $2 \mathrm{D} / 3 \mathrm{D}$ era.

Hence these historical reasons apply only to a certain extent in the modern-day radiation oncology. CSI targets all of the leptomeningeal metastatic disease, macroscopic and microscopic. Patients receiving CSI do not have a risk of developing radiculitis, meningitis, and several other side effects and they are not subjects of repeated lumbar punctures which are needed to apply IT chemotherapy and are themselves painful and hence decrease the quality of life.

\section{Limitations}

The major limitations of our study was its retrospective nature, the patients' selection bias, and small and heterogeneous patients' collective. All patients were treated before 2016 and none of the patients received small molecules, TKIs or immune checkpoint inhibitors prior to the treatment with CSI or thereafter. However, these of novel agents are used more frequently today may have an impact on oncologic outcome after CSI. Furthermore, due to the retrospective design, there is a potential selection bias regarding which patients have received a CSI.

\section{Conclusion}

CSI demonstrated clinically meaningful survival that is comparable to the reported outcome of intrathecal chemotherapy. A simple scoring system could be used to better select patients for treatment with CSI in the palliative setting. Especially in patients with larger macroscopic lesions, the benefit of RT is undisputed. In our opinion, the feasibility of performing CSI with modern radiotherapy techniques with better sparing of healthy tissue gives a further rationale for its use also in the palliative setting.

\section{Abbreviations \\ LM: leptomeningeal metastases; NSCLC: non-small cell lung cancer; NCCN: National comprehensive cancer network; KPS: Karnofsky performance score; IT: intrathecal; HD-MTX: high dose methotrexate; WBRT: whole brain radiotherapy; RT: radiotherapy; ESMO: European society for medical oncology; CSI: craniospinal irradiation; IMRT: intensity modulated radiotherapy; PNET: primitive neuroectodermal tumor; SI: spinal irradiation; HT: helical tomotherapy; OAR: organs at risk; CTV: clinical target volume.; PTV: planning target volume; OS: overall survival}

\section{Acknowledgements}

Not Applicable.

\section{Authors' contributions}

Conceived and designed the study: MD, MND, SEC. Analyzed the data: MD; MND; JJW; SK; SM; CS; SEC. Contributed materials/analysis tools: MD. Wrote the paper: MD; MND; JJW; SK; SM; CS; KJB; SEC. All authors read and approved the final manuscript.

\section{Funding}

Not applicable.

\section{Availability of data and materials}

The datasets used and/or analyzed during the current study are available from the corresponding author on reasonable request.

\section{Ethics approval and consent to participate}

The Ethic committee of the Technical University Munich has approved this retrospective study (number 84/15). All patients gave their informed written consent for radiotherapy. The need for a second informed consent for his retrospective study was deemed unnecessary. 


\section{Competing interests}

MD - Wife employed at Brainlab.

SK- received speaker honoraria from Accuray.

CS- received a scholarship from Medac $\mathrm{GmbH}$; received a travel grant from NovoCure Ltd.; contributed to a brochure for patients about GBM which was partially sponsored by NovoCure, received speakers Honoria from Teva $\mathrm{GmbH}$.

NMD: editor at BMC

SEC: Advisory Boards/Advisor: BMS, Astra Zeneca, Roche, Novocure, Daiichi Sankyo, Icotec; Speakers Honoraria: BMS, Astra Zeneca, Roche, Novocure, Daiichi Sankyo, Icotec, Brainlab, varian, Accuray, Zeiss Meditec, Dr. Sennewald, Elekta, Merck Darmstadt, Medac.

All other authors have nothing to disclose.

\section{Author details}

'Klinikum rechts der Isar, Department of RadiationOncology, Technical University Munich, Ismaninger Strasse 22, 81675 München, Germany. ${ }^{2}$ Department of Radiotherapy and Radiation Oncology, University Hospital of the Friedrich Schiller University, Jena, Germany. ${ }^{3}$ Deutsches Konsortium für Translationale Krebsforschung (DKTK)-Partner Site Munich, Munich, Germany. ${ }^{4}$ Institute of Innovative Radiotherapy, Helmholtzzentrum München, Munich, Germany.

Received: 4 September 2019 Accepted: 20 May 2020

Published online: 01 June 2020

\section{References}

1. Chamberlain MC. Neoplastic meningitis. J Clin Oncol. 2005;23(15):3605-13.

2. Leal T, Chang JE, Mehta M, Robins HI. Leptomeningeal metastasis: challenges in diagnosis and treatment. Curr Cancer Ther Rev. 2011;7(4):319-27.

3. Wasserstrom WR, Glass JP, Posner JB. Diagnosis and treatment of leptomeningeal metastases from solid tumors: experience with 90 patients. Cancer. 1982;49(4):759-72.

4. Gleissner B, Chamberlain MC. Neoplastic meningitis. Lancet Neurol. 2006; 5(5):443-52.

5. Hollender A, Kvaloy S, Nome O, Skovlund E, Lote K, Holte H. Central nervous system involvement following diagnosis of non-Hodgkin's lymphoma: a risk model. Ann Oncol. 2002;13(7):1099-107.

6. NCCN. NCCN Guidelines Version 01.2017 Central Nervous System Cancers. NCCN Guidelines. 2017;01.2017. https:/www.nccn.org/professionals/ physician_gls/pdf/cns.pdf. Accessed 4 Sept 2017.

7. Le Rhun E, Weller M, Brandsma D, et al. EANO-ESMO Clinical Practice Guidelines for diagnosis, treatment and follow-up of patients with leptomeningeal metastasis from solid tumours. Ann Oncol. 2017;28(suppl_ 4):iv84-99.

8. Sugie C, Shibamoto Y, Ayakawa S, et al. Craniospinal irradiation using helical tomotherapy: evaluation of acute toxicity and dose distribution. Technol Cancer Res Treat. 2011;10(2):187-95

9. Kusters JM, Louwe RJ, van Kollenburg PG, et al. Optimal normal tissue sparing in craniospinal axis irradiation using IMRT with daily intrafractionally modulated junction(s). Int J Radiat Oncol Biol Phys. 2011;81(5):1405-14.

10. Bandurska-Luque A, Piotrowski T, Skrobala A, Ryczkowski A, Adamska K, Kazmierska J. Prospective study on dosimetric comparison of helical tomotherapy and 3DCRT for craniospinal irradiation - a single institution experience. Rep Pract Oncol Radiother. 2015;20(2):145-52.

11. Weller M. Metastasen und Meningeosis neoplastica. 2014. http://www. neuroonkologie.de/files/guidelines/7-spinale-metastasen-und-meningeosisneopl.pdf. Accessed 6.9.2017.

12. Pan Z, Yang G, He H, et al. Concurrent radiotherapy and intrathecal methotrexate for treating leptomeningeal metastasis from solid tumors with adverse prognostic factors: a prospective and single-arm study. Int I Cancer. 2016;139(8):1864-72.

13. Rudnicka $\mathrm{H}$, Niwinska A, Murawska M. Breast cancer leptomeningeal metastasis--the role of multimodality treatment. J Neuro-Oncol. 2007;84(1): 57-62.

14. Boogerd W, van den Bent MJ, Koehler PJ, et al. The relevance of intraventricular chemotherapy for leptomeningeal metastasis in breast cancer: a randomised study. Eur J Cancer. 2004;40(18):2726-33.

15. Oechsle K, Lange-Brock V, Kruell A, Bokemeyer C, de Wit M. Prognostic factors and treatment options in patients with leptomeningeal metastases of different primary tumors: a retrospective analysis. J Cancer Res Clin Oncol. 2010;136(11):1729-35.

16. Herrlinger $\mathrm{U}$, Forschler $\mathrm{H}$, Kuker $\mathrm{W}$, et al. Leptomeningeal metastasis: survival and prognostic factors in 155 patients. J Neurol Sci. 2004;223(2):167-78.

17. Rogers $L R$, Remer $S E$, Tejwani $S$. Durable response of breast cancer leptomeningeal metastasis to capecitabine monotherapy. Neuro-Oncology. 2004;6(1):63-4.

18. Tham YL, Hinckley L, Teh BS, Elledge R. Long-term clinical response in leptomeningeal metastases from breast cancer treated with capecitabine monotherapy: a case report. Clin Breast Cancer. 2006;7(2):164-6.

19. Ekenel M, Hormigo AM, Peak S, Deangelis LM, Abrey LE. Capecitabine therapy of central nervous system metastases from breast cancer. J NeuroOncol. 2007;85(2):223-7.

20. Hermann B, Hultenschmidt B, Sautter-Bihl ML. Radiotherapy of the neuroaxis for palliative treatment of leptomeningeal carcinomatosis. Strahlenther Onkol. 2001;177(4):195-9.

21. El Shafie RA, Bohm K, Weber D, et al. Outcome and prognostic factors following palliative craniospinal irradiation for leptomeningeal carcinomatosis. Cancer Manag Res. 2019;11:789-801.

22. Barney CL, Brown AP, Grosshans DR, et al. Technique, outcomes, and acute toxicities in adults treated with proton beam craniospinal irradiation. NeuroOncology. 2014;16(2):303-9.

23. Brown AP, Barney $C L$, Grosshans DR, et al. Proton beam craniospinal irradiation reduces acute toxicity for adults with medulloblastoma. Int J Radiat Oncol Biol Phys. 2013;86(2):277-84.

24. Petersson K, Gebre-Medhin M, Ceberg C, et al. Haematological toxicity in adult patients receiving craniospinal irradiation--indication of a dose-bath effect. Radiother Oncol. 2014;111(1):47-51.

25. Parker W, Filion E, Roberge D, Freeman CR. Intensity-modulated radiotherapy for craniospinal irradiation: target volume considerations, dose constraints, and competing risks. Int J Radiat Oncol Biol Phys. 2007;69(1): 251-7.

\section{Publisher's Note}

Springer Nature remains neutral with regard to jurisdictional claims in published maps and institutional affiliations.

Ready to submit your research? Choose BMC and benefit from:

- fast, convenient online submission

- thorough peer review by experienced researchers in your field

- rapid publication on acceptance

- support for research data, including large and complex data types

- gold Open Access which fosters wider collaboration and increased citations

- maximum visibility for your research: over $100 \mathrm{M}$ website views per year

At $\mathrm{BMC}$, research is always in progress.

Learn more biomedcentral.com/submissions 